\title{
Is it what you see, or how you say it? Spatial bias in young and aged subjects
}

\author{
ANNA M. BARRETT ${ }^{1-3}$ AND CATHERINE E. CRAVER-LEMLEY ${ }^{4}$ \\ ${ }^{1}$ Kessler Medical Rehabilitation Research and Education Center, West Orange, New Jersey, \\ ${ }^{2}$ Department of Physical Medicine and Rehabilitation, New Jersey Medical School, University of Medicine and Dentistry, \\ New Jersey-Newark, New Jersey \\ ${ }^{3}$ Department of Neurology and Neurosciences, New Jersey Medical School, University of Medicine and Dentistry, \\ New Jersey-Newark, New Jersey \\ ${ }^{4}$ Department of Psychology, Elizabethtown College, Elizabethtown, Pennsylvania
}

(Received February 7, 2007; Final Revision March 13, 2008; AcCePted March 14, 2008)

\begin{abstract}
Healthy subjects demonstrate leftward bias on visual-spatial tasks. However, young controls may also be left-biased when drawing communicatively, depicting the subject of a sentence leftward on a page relative to the sentence object, that is, a spatial-syntactic, implicit task. A leftward visual-spatial bias may decrease with aging, as right-hemisphere, dorsal, visual-spatial activation may be reduced in elderly subjects performing these tasks. We compared horizontal and radial (near-far) visual spatial bias, and spatial-syntactic bias, in healthy young and aged participants. Both horizontal and radial visual-spatial bias were smaller in aged participants when explicitly, but not implicitly assessed. Mean implicit far bias was greater in aged subjects, although this varied by task. We observed less implicit, spatial-syntactic left bias in aged than young participants. These results may be consistent with relatively less dominance of right hemisphere, dorsal spatial systems with aging. (JINS, 2008, 14, 562-570.)
\end{abstract}

Keywords: Visual attention, Spatial function, Line bisection, Aging, Perception, Body space

\section{INTRODUCTION}

Healthy control groups may demonstrate leftward bias when marking the center of a line or a space (Jewell \& McCourt, 2000; Tegner \& Levander, 1991). Leftward errors might be attributed to greater activation in right versus left cortical hemispheric systems, as subjects perform this explicit, visual-spatial coordinate computation.

When stimuli or task demands are changed in visual picture manipulation, however, we might expect asymmetric hemispheric activation to change as well. Object naming, detection tasks (Previc \& Blume, 1993; Reuter-Lorenz \& Posner, 1990), tasks in which spatial stimuli are categorized (Kosslyn et al., 1989), and tasks dependent on linguistic processing (e.g., Jordan \& Patching, 2003) may be associated with rightward bias. It may thus be surprising that, when asked to depict the action of a sentence in a

Correspondence and reprint requests to: Anna M. Barrett, MD, Kessler Medical Rehabilitation Research and Education Center, 1199 Pleasant Valley Way, West Orange, NJ 07052. E-mail: abarrett@kmrrec.org drawing, groups of healthy young participants show a robust tendency to draw the subject of a sentence placed leftward on the paper relative to the sentence object. Although this task requires syntactic representations and thus is communicative, it may tap a more fundamental form of grammatic depiction than the mature left hemisphere speech and language system (see reviews in Barrett et al., 2002a; cf. Chatterjee et al., 1995; Chatterjee, 2001; Geminiani et al., 1995). We previously investigated this spatial-syntactic bias in Korean people who originally learned right to left, vertical reading, but did not find it to differ from that of culturally matched individuals who learned to read left to right and horizontally (Barrett et al., 2002a).

The spatial-syntactic task is different from tasks assessing visual-spatial computations, such as bisecting a line, because of the nature of its stimuli. However, when subjects depict sentences in drawings, they are also not aware that placement of the drawings on the paper will be assessed. Any visual-motor task requires the coordination of bihemispheric and disparate brain systems. Thus, implicitly assessing spatial bias may make a contributing or underlying 
spatial bias manifest, which might otherwise be obscured by coexisting linguistic or object processing. Although implicit tasks are commonly used to assess cognition, most paper and pencil visual-motor spatial tasks assess performance explicitly. This is ironic, because the "where" operations of the dorsal visual system associated with visualspatial function (Ungerleider \& Mishkin, 1982) are largely implicit, inaccessible to consciousness monitoring (Milner \& Goodale, 1996).

Jewell and McCourt (2000) summarized previous reports and concluded that aged people may demonstrate less leftward bias than young subjects on the visual-spatial, line bisection task. There are at least two possible explanations for agerelated changes in line bisection bias. (1) Aging may be associated with less obligate activity in right hemisphere dorsal "where" visual-spatial systems as the subjects process line stimuli and prepare a motor response (Weiss et al., 2003). Different effects of aging on cognitive systems differentially lateralized in right versus left cortical hemispheres may underlie this change (e.g., Dolcos et al., 2002; Harvey \& Butler, 2004), and aging may adversely affect right hemisphere dorsal spatial systems more than it affects left hemisphere dorsal systems. Hemispheric asymmetry may thus be reduced with aging for spatial tasks as it may be on verbal memory and other cognitive tasks (the HAROLD model; Cabeza, 2002). (2) It is also possible, however, that decreased bias on the line bisection task actually reflects increased task accuracy with aging (smaller magnitude error).

One means of distinguishing spatial performance changes with aging due to changes in brain localization of function, from changes in bias due to increased task accuracy, is to examine spatial bias along more than one axis. When bisecting a radial line that lies within reaching distance and extends in the midline body mid-sagittal plane at a 90-degree angle with body vertical (a near-far axis such as might be relevant to paper and pencil tasks presented on a tabletop), healthy young controls consistently mark the line's center distal to its veridical midline (far line bisection errors; e.g., Barrett et al., 2002b). This bias is observed even in subjects with spatial neglect after right hemisphere injury (e.g., Mark \& Heilman, 1998) and so may not be critically dependent on right hemisphere dorsal brain function. If dorsal-stream spatial brain systems are strongly activated during line bisection, but with aging this is less right hemisphere-specific, left spatial bias on this task may decrease, but a far spatial bias should be maintained. Far bias might even increase in aged over young subjects, as left hemisphere, dorsal systems may have an affinity for far as compared with near space operations (Previc, 1990; Varnava et al., 2002). If altered horizontal line bisection accuracy in aged as compared with young subjects is not explained by changes in hemispheric dominance in dorsal spatial brain systems, however, aged subjects might make smaller errors on radial (near-far) line bisection compared with young subjects.

There is a second means of determining whether decreased leftward line bisection errors in aged subjects may be due to decreased hemispheric asymmetry in spatial processing systems, rather than changes in task accuracy. One could assess leftward bias in aged versus young subjects with an implicit task, in which subjects are not aware of, or attempting to meet, a performance standard. Such a task might reduce differences in performance characteristics related to self-monitoring and conscious strategies, which may occur with aging. Assessing spatial syntactic bias by asking subjects to draw an object or depict the action of a simple subject-verb-object sentence is such a task. Subjects must manifest bias on this testing (must choose horizontal and radial coordinates to center the object on the paper, and are forced to place the subject either to the left or the right of the object for each trial), but neither drawing an object leftward of center or rightward of center, or placing a sentence subject on the left or right of the object, is by definition "correct."

In this study, we sought to learn whether, if a leftward visual-spatial bias decreases with aging, there is also a decrease in radial (far) spatial bias. We wished to examine horizontal and radial visual-spatial bias both explicitly (by testing line bisection), and implicitly (by having subjects draw objects, and then examining how they centered their drawings on the paper). If performance accuracy changes occur because of differences in conscious strategies with aging, this might be expected to influence explicit errors, but not implicit spatial bias.

We also examined implicit leftward visual-spatial and spatial-syntactic bias in aged versus young subjects performing drawing tasks. In the implicit visual-spatial task, the coordinates at which subjects centered an object were assessed (house/tree/person drawing; Heller, 1991). In the implicit spatial-syntactic task, we examined placement of drawn elements of a dictated sentence (cf. Chatterjee et al., 1995; Geminiani et al., 1995). In a previous study of Korean subjects, we observed that implicit visual-spatial bias was increased in aged versus young subjects, but implicit leftward spatial-syntactic bias was not observed in either aged or young subjects (Barrett et al., 2002a). However, aged and young Korean subjects in that study had different initial learned reading exposure, and both had been exposed to right-to-left reading.

In this study, we examined Western subjects who all learned left-to-right, horizontal reading of the English language. Parallel decreases in spatial-syntactic and visualspatial bias might suggest distributed changes occur in right hemisphere, dorsal "where" systems with aging, affecting both coordinate and categorical "where" spatial systems (Kosslyn et al., 1989).

\section{METHOD}

The logic of the study, and some major results and conclusions, is diagrammed in Figure 1 and further detailed in the Discussion section. All data in this study were collected in compliance with the regulations of our institutions, and in accordance with the guidelines of the Helsinki Declaration. 


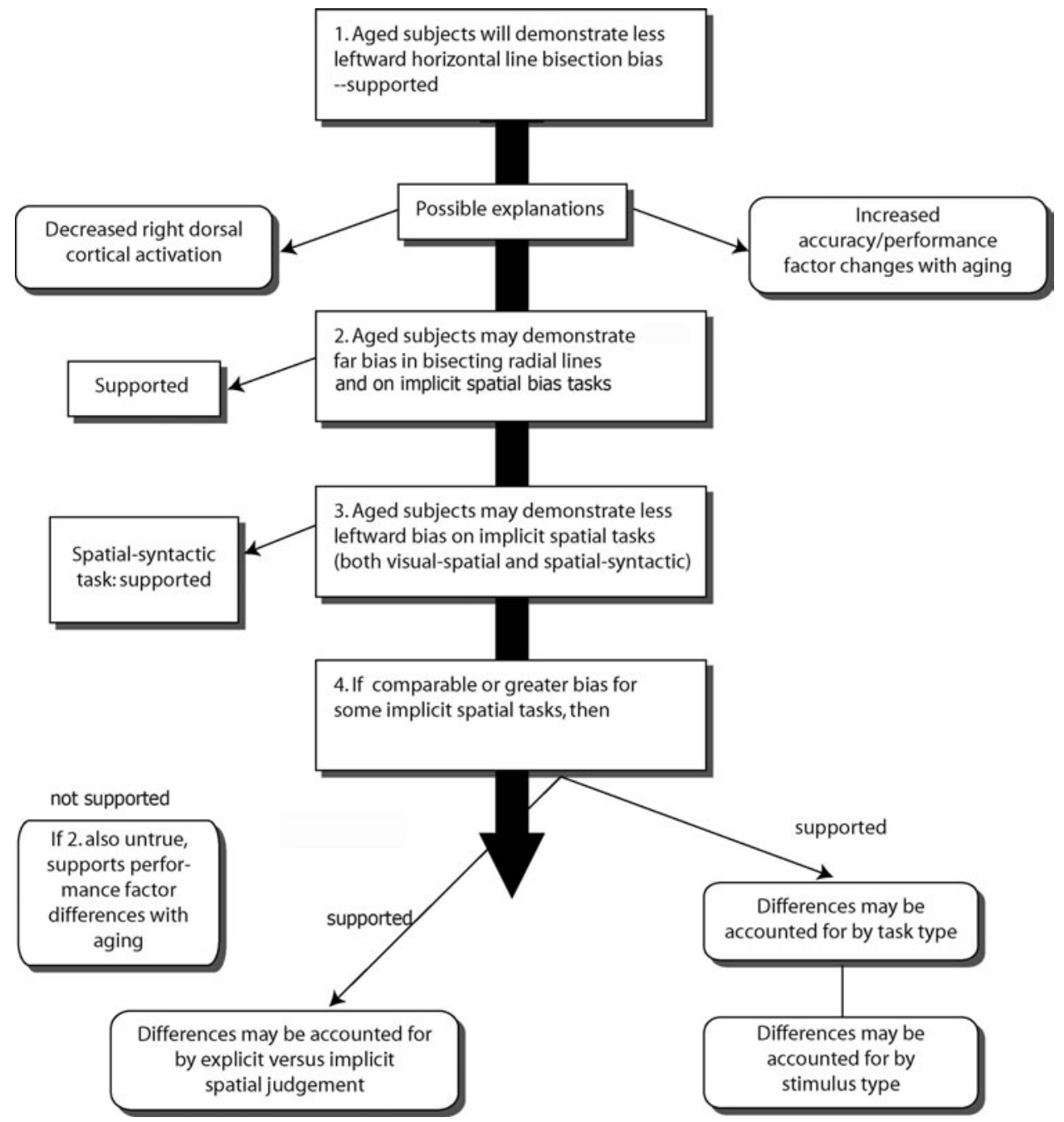

Fig. 1. Logic of the theoretical approach and reasoning for the current study. Please see the text for detail. Arrows indicate inferences supported by the data presented.

\section{Subjects}

We tested 60 young (mean age, 20.4 years; $S D$ 2.03; 30 women) and 60 aged (mean age, 73.7 years; $S D$ 5.78; 30 women) participants on four paper-and-pencil tasks. All subjects were without neurological or psychiatric history, and aged subjects scored a mean 28.6 of 30 (range, 26-30; SD 1.22) on the Mini-Mental State Examination (Folstein et al., 1975). All of the data included in the current research was obtained in compliance with our institutional regulations. The tasks described before have been previously used, and results reported, in a Korean subject group (Barrett et al., 2002a).

\section{Visual-Spatial Tasks: Line Bisection}

Subjects bisected five $22-\mathrm{cm}$ horizontal lines and five $24-\mathrm{cm}$ horizontal lines presented on separate pieces of paper. We included two horizontal and radial line lengths, because small stimulus changes enhance subjects' attention to performing the task consistently, and thus promote validity of collected data. Subjects bisected five 16-cm radial lines and five $18-\mathrm{cm}$ radial lines, all in the mid-sagittal plane. Lines were printed $1 \mathrm{~mm}$ thick and centered on white standard $21.6 \mathrm{~cm} \times 27.9 \mathrm{~cm}(8.5 \times 11 \mathrm{inch})$ paper, oriented with long edge horizontal and centered with respect to their bodies on a table approximately $30 \mathrm{~cm}$ from their eyes. Errors were measured in millimeters, and coded positive if rightward of (horizontal lines) or distal to (radial lines) line center, negative if leftward of (horizontal lines) or proximal to (radial lines) line center. For each subject, we calculated mean horizontal line bisection bias over both line lengths (total error/10) and mean radial line bisection bias over both line lengths (total error/10).

\section{Visual-Spatial Tasks: Drawing Placement}

Subjects were tested on the house/tree/person drawing task (Buck, 1966). Each subject was instructed to draw sequentially a house, a tree, and a person, on three separate standard sheets of $8.5^{\prime \prime} \times 11^{\prime \prime}$ paper with long edge oriented 
horizontally and centered with respect to their bodies approximately $30 \mathrm{~cm}$ from their eyes. Although this task is traditionally used as a psychological projective test, we used it to assess implicit visual-spatial bias (Heller, 1991). We assessed horizontal displacement of each drawing from the center of the page by measuring the distance in millimeters from the midpoint of the horizontal extent of each picture to the horizontal midpoint of the paper. We similarly measured radial (near/far) displacement of each drawing from the center of the page, by measuring the distance in $\mathrm{mm}$ from the midpoint of the radial extent of each picture to the radial midpoint of the paper. Rightward displacement and far displacement was coded positive and leftward displacement and near displacement, negative.

\section{Spatial-Syntactic Task}

Subjects were asked to draw, on a standard $8.5^{\prime \prime} \times 11^{\prime \prime}$ sheet of paper with long edge oriented horizontally, four sentences, which the examiner read aloud. Each sentence was read aloud just before it was drawn, and repeated in its entirety as many times as the subject requested. The four sentences were: "A man shoots a gun at a fierce tiger," "A father scolds his naughty child," "A woman chases her dog, who has broken his leash," and "A mother combs her child's hair." Subjects were not given any feedback about their drawings, but were urged to include all the sentence elements. See Figure 2 for examples of drawings produced for this task.

We wished to assess subjects' tendency to draw the subject of the sentence placed so that it was positioned leftward on the paper relative to the position of the object of the sentence. A spatial-syntactic bias ratio was thus calculated for each subject by the following formula:

(\# of drawings with subject on the right) - (\# of drawings with subject on the left)

4

This yields a number between -1 and +1 , with -1 indicating that in all four drawings the subject was placed to the left of the object of the sentence, and +1 indicating that in all four drawings the subject was placed to the right of the object of the sentence. An intermediate value indicates that the subject of the sentence may have been placed in a different position relative to the object in one or more of the drawings.

\section{Data Analysis}

We tested four, a priori, hypotheses. (See Figure 1, which diagrams this logic and some of the pertinent study results.)

(1) We hypothesized that we would replicate reduced line bisection bias in aged compared with young subjects. We also hypothesized that aged subjects might not make significant leftward line bisection errors

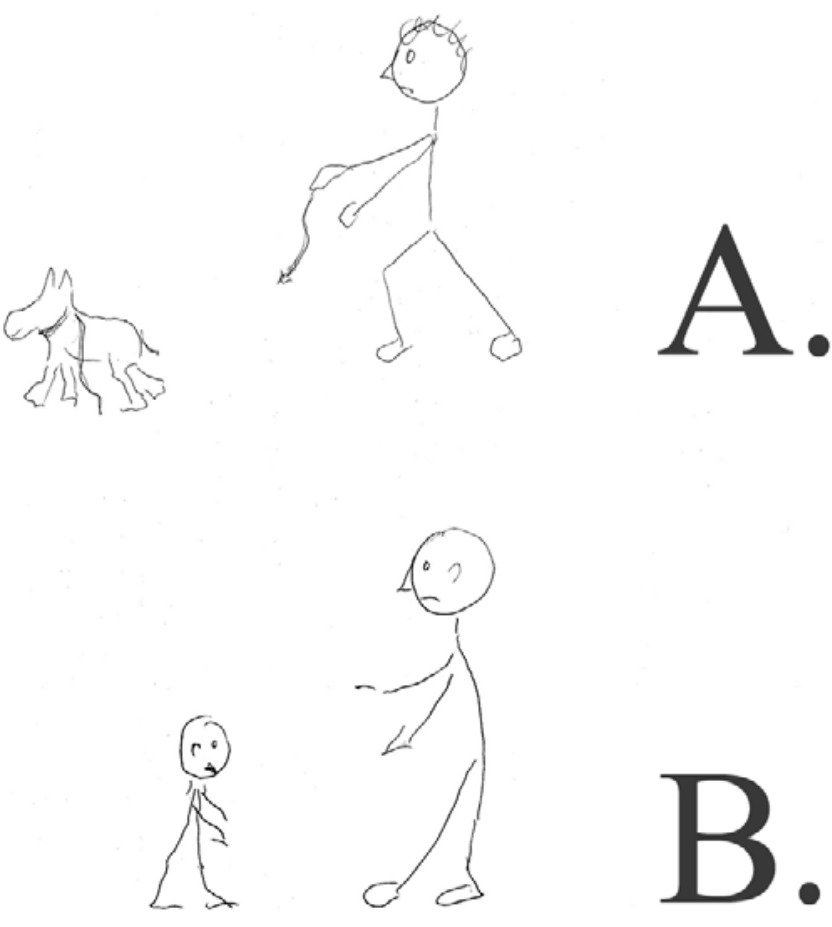

Fig. 2. Examples of drawings produced by an aged subject in the current experiment, intended to illustrate, "A woman chases her dog, who has broken his leash" (A) and "A father scolds his naughty child" (B). In these productions, the sentence subject (a woman, a father) was depicted on the right side of the object (her dog, his child).

when compared with perfect performance, but that young subjects would make significant leftward errors. To test these a priori hypotheses, we performed an independent-samples Student's $t$ test comparing mean horizontal line bisection errors (over both $22-\mathrm{cm}$ and $24-\mathrm{cm}$ lines) between young and aged subject groups. We then performed one-sample $t$ tests comparing mean horizontal line bisection error to zero (perfect performance), separately for the young and aged groups.

(2) We hypothesized that radial line bisection bias might be reduced in aged versus young subjects if a reduction in errors was primarily related to the task rather than brain lateralization of spatial function. Aged subjects might then also not make significant far line bisection errors when compared with perfect performance, but young subjects should make significant far line bisection errors. To test these a priori hypotheses, we performed an independent samples $t$ test comparing mean radial line bisection errors (over both $16-\mathrm{cm}$ and $18-\mathrm{cm}$ line lengths) between young and aged subject groups. We then performed onesample $t$ tests comparing mean horizontal line bisection error to zero (perfect performance) separately for the young and aged groups.

(3) We hypothesized that, if performance characteristics or conscious strategy reduced line bisection error in 
Table 1. Subjects and results for the current experiment

\begin{tabular}{|c|c|c|c|}
\hline & $\begin{array}{c}\text { Younger subjects } \\
(n=60 ; 30 \text { women })\end{array}$ & $\begin{array}{c}\text { Aged subjects } \\
(n=60 ; 30 \text { women })\end{array}$ & $p$ value $^{\mathrm{a}}$ \\
\hline Age (mean years) $\pm S D$ & $20.4 \pm 2.03$ & $73.7 \pm 5.78$ & - \\
\hline Education (mean years) $\pm S D$ & $14.6 \pm 1.66$ & $14.4 \pm 2.87$ & n.s. \\
\hline Horizontal line bisection error (in $\mathrm{mm}$ ) $\pm S D$ & $\begin{array}{c}-2.6 \mathrm{~mm}(\text { leftward }) \pm 2.80 \\
p<.001\end{array}$ & $\begin{array}{c}-0.8 \mathrm{~mm}(\text { leftward }) \pm 4.40 \\
p<.17\end{array}$ & .008 \\
\hline Radial line bisection error (in $\mathrm{mm}$ ) $\pm S D$ & $\begin{array}{c}3.8 \mathrm{~mm}(\text { distal }) \pm 2.91 \\
p<.001\end{array}$ & $\begin{array}{l}2.4 \mathrm{~mm}(\text { distal }) \pm 4.24 \\
p<.001\end{array}$ & .048 \\
\hline Horizontal drawing displacement (in $\mathrm{mm}) \pm S D$ & $\begin{array}{c}-5.0 \mathrm{~mm}(\text { distal }) \pm 17.33 \\
p<.025\end{array}$ & $\begin{array}{c}-8.2 \mathrm{~mm}(\text { distal }) \pm 15.88 \\
p<.005\end{array}$ & .288 \\
\hline Radial drawing displacement (in $\mathrm{mm}$ ) $\pm S D$ & $\begin{array}{c}-2.9 \mathrm{~mm} \text { (proximal) } \pm 13.42 \\
p<.22\end{array}$ & $\begin{array}{l}5.0 \mathrm{~mm}(\text { distal }) \pm 19.48 \\
p<.005\end{array}$ & .011 \\
\hline Spatial-syntactic ratio $\pm S D$ & $\begin{array}{c}-0.7 \text { (leftward) } \pm 0.53 \\
p<.001\end{array}$ & $\begin{array}{c}-0.4 \text { (leftward) } \pm 0.82 \\
\quad p<.003\end{array}$ & .045 \\
\hline
\end{tabular}

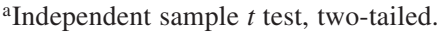

either the horizontal or radial directions in aged subjects, this might not occur for implicit tasks. Strategy may have less effect on implicit behaviors, which are not consciously monitored. To test this a priori hypothesis, we performed the same comparisons as noted above (independent-samples $t$ tests comparing mean horizontal displacement over the three drawings, and mean radial displacement over the three drawings, between age groups; one-sample $t$ tests comparing horizontal displacement in young and aged groups to zero, and comparing radial displacement in young and aged groups to zero). Radial displacement was not assessed in the implicit sentence drawing task.

(4) We hypothesized that, if reduced hemispheric laterality of right hemisphere-mediated, dorsal spatial function underlies a reduction in leftward line bisection bias, other spatial biases reliant on this system might also be reduced. We previously observed that aged subjects failed to manifest leftward bias on a spatial-syntactic task (Barrett et al., 2002a), but these groups of Korean subjects learned to read left-toright. To test the a priori hypotheses that spatialsyntactic bias might be reduced in aged versus young subjects, and might be absent in the aged subject group while present in the young subjects, we performed a Mann-Whitney $U$ test comparing the number of drawings with the subject placed on the left, and the number with the subject placed on the right, over the young and aged subject groups. Within each age group, we then sought a manifest leftward spatial syntactic bias by comparing spatial syntactic bias ratio with zero with a Wilcoxon Signed Ranks Test.

Although some a priori hypotheses were directional as above, significance levels reported are those for two-tailed comparisons. Appropriate evaluation of homogeneity of vari- ance was carried out for each parametric comparison using Levene's test or Maunchly's test of sphericity for equality of variance, as appropriate. Where these evaluations indicated inhomogeneity of variance, we made no assumption of homogeneity and report $p$ values accordingly.

\section{RESULTS}

\section{Hypothesis 1}

Absence of horizontal line bisection bias in aged as compared with young subjects (see Table 1 for summary). Aged subjects made a mean $-0.8 \mathrm{~mm}$ leftward line bisection error ( $S D$ 4.40), significantly less compared with young subjects' mean $-2.6 \mathrm{~mm}$ leftward line bisection error $(S D=$ $2.80 ; t=2.72 ; p=.008)$. Although leftward line bisection errors reached significance in young subjects $(t=-7.26$; $p<.001$ ), they did not reach significance in aged subjects $(t=1.41 ; p=.17$; n.s. $)$.

\section{Hypothesis 2}

Reduction in radial line bisection bias with aging. When far line bisection error in aged subjects, a mean $2.4 \mathrm{~mm}$ distal to veridical center ( $S D 4.24)$, were compared with a larger magnitude far line bisection error in young subjects, a mean $3.8 \mathrm{~mm}$ distal to veridical center ( $S D$ 2.91), this difference was significant $(t=-2.01 ; p=.048)$. Far line bisection errors, however, reached significance in both young subjects $(t=3.82 ; p<.001)$ and their aged counterparts $(t=$ $3.89 ; p<.001)$. See Figure 3 for illustration of findings relevant to Hypotheses 1 and 2.

\section{Hypothesis 3}

No reduction in implicit visual-spatial bias with aging. A mean leftward bias in placing house/tree/person drawings 


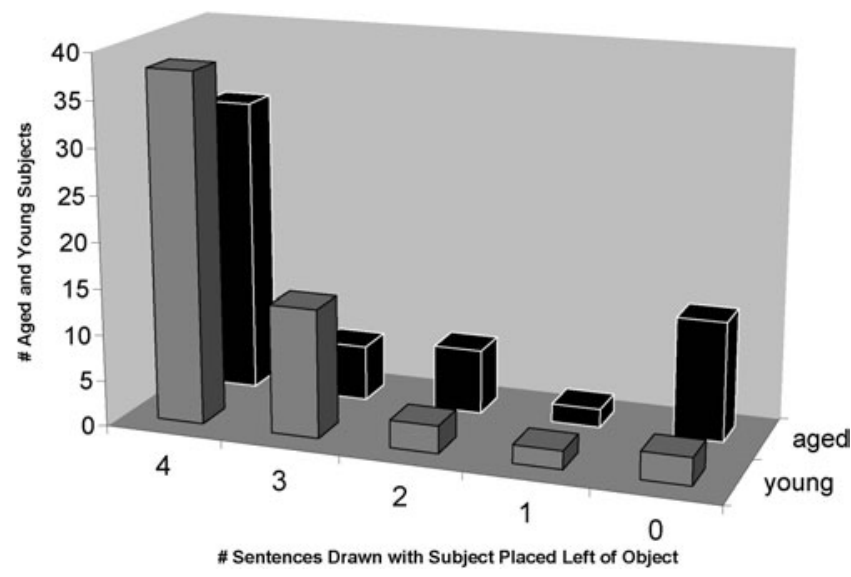

Fig. 3. Results of explicit and implicit visual-spatial bias assessment with line bisection and drawing placement tasks, in 120 young and aged subjects. Millimeters of mean error are depicted on the $x$-axis (negative error indicates leftward bias for horizontal line bisection, and proximal/near bias for radial line bisection). Thin lines depict standard deviation. Aged subjects had no mean leftward error on horizontal line bisection (black bars), while young subjects (gray bars) erred leftward (***group difference where $p<.01$, please see text for details). Aged subjects and young subjects both made distal (far) radial line bisection errors, greater in magnitude in young subjects (*group difference where $p<$ $.05)$. For implicit, drawing placement, tasks (horizontal pictures, radial pictures); however, aged subjects made larger magnitude leftward and distal/far errors than did young subjects (*group difference where $p<.05$, please see text for details).

on the page relative to actual page center in aged subjects $(-8.3 \mathrm{~mm}$ leftward of page center; $S D$ 15.87) did not differ significantly from a mean leftward bias in young subjects ( $-5.0 \mathrm{~mm}$ leftward; $S D$ 17.33; $t=-1.07 ; p=.288$; n.s.). Leftward bias in both aged and young subjects was significantly different from zero (one-sample $t$ tests; aged: $t=$ $-4.02 ; p<.001$; young: $t=-2.23 ; p=.029$ ). Aged subjects actually demonstrated larger magnitude implicit far bias, placing drawings further distal to actual page center (mean $5.0 \mathrm{~mm}$ distal to page center; $S D$ 13.42) than did young subjects (mean $-2.9 \mathrm{~mm}$ proximal to center, $S D$ 19.48; $t=2.60 ; p=.011$ ), although only the far bias in aged subjects reached significance when compared with zero (one-sample $t$ tests; aged: $t=2.89 ; p=.005$; young: $t=-1.17 ; p=.25 ;$ n.s.). See Figure 3 for illustration of this finding.

\section{Hypothesis 4}

Reduction in magnitude of spatial-syntactic bias in aged as compared with young subjects. The spatial-syntactic bias ratio in aged subjects (mean $-0.4 ; S D 0.83$ ) was less leftward than that for young subjects (mean $-0.7 ; S D 0.53 ; z=$ $-2.00 ; p<.05$; two-tailed). However, both aged and young subjects had leftward spatial-syntactic bias when the spatialsyntactic bias ratio was compared with zero (aged: $z=$
$-2.94 ; p=.003$; young: $z=-5.91 ; p<.001)$. See Figure 4 for an illustration of this finding.

\section{Post hoc Analyses}

\section{(A) Aged subjects not more variable than young subjects at line bisection}

We observed that mean error was smaller in aged subjects bisecting horizontal and radial lines than mean error at the same tasks performed by young subjects. This might mean that their performance was more accurate. However, it is possible that a greater variability of performance in aged subjects may have occurred across the veridical line center, giving rise to smaller magnitude mean signed errors. This greater variability is not consistent with more accurate performance. To examine the post hoc hypothesis that greater variability accounted for smaller magnitude mean signed error, we calculated the standard deviation for mean horizontal and radial line bisection errors for each subject. We then compared the standard deviation for $22-$ and $24-\mathrm{cm}$ horizontal and 16- and $18-\mathrm{cm}$ radial line bisections error between young and aged subjects using four separate independent-samples $t$ tests.

Differences in the standard deviation of each subject's line bisection performance did not reach significance across old and young subject groups for 22-cm (mean aged = $2.70 \mathrm{~mm}$; mean young $=2.71 \mathrm{~mm} ; p=.95 ; \mathrm{n} . \mathrm{s}$.); or $24-\mathrm{cm}$ horizontal lines (mean aged $=2.90 \mathrm{~mm}$; mean young $=$ $2.83 \mathrm{~mm} ; p=.77$; n.s.); nor did these differences reach significance for $16-\mathrm{cm}$ (mean aged $=2.12 \mathrm{~mm}$; mean young $=2.14 \mathrm{~mm} ; p=.90$; n.s.) or 18 -cm radial lines (mean aged $=2.22 \mathrm{~mm}$; mean young $=2.47 \mathrm{~mm} ; p=.22$; n.s.). This suggests that aged subjects were not more variable in their performance than were young subjects.

\section{(B) Differences by subject content of house/tree/person drawings}

What the subjects drew (a house, tree, or person) did not appear to influence horizontal left/right bias, but did affect radial near/far bias. Aged and young subjects both centered drawings leftward when drawing a "house" (aged mean $-9.85 \mathrm{~mm}$; $S D$ 19.50; young mean $-5.67 \mathrm{~mm}$; $S D$ 19.95; $t=-1.15, p=.25$; n.s.), a "tree" (aged mean $-6.41 \mathrm{~mm}$; $S D$ 21.06; young mean $-5.83 \mathrm{~mm} ; S D$ 19.95; $t=-0.16$, $p=.88$; n.s.), and a "person" (aged mean $-8.83 ; S D$ 17.18; young mean $-3.35 \mathrm{~mm} ; S D 19.95 ; t=-1.61, p=.11$; n.s.). However, both aged and young subjects centered "house" drawings distal to radial center (aged $8.20 \mathrm{~mm} ; S D$ 18.54; young mean $1.98 \mathrm{~mm} ; S D 23.38 ; \mathrm{t}=1.61 ; p=.11$; n.s.). Neither age group centered their "person" drawings distally (aged mean $-1.38 \mathrm{~mm}$; $S D$ 19.17; young mean $-10.19 \mathrm{~mm}$; $S D$ 22.97), and age group comparison did not reach significance after Bonferroni correction $(t=2.28$; $p=.024)$. However, aged subjects centered their "tree" drawings distally (mean $8.16 \mathrm{~mm} ; S D$ 14.99), while young 


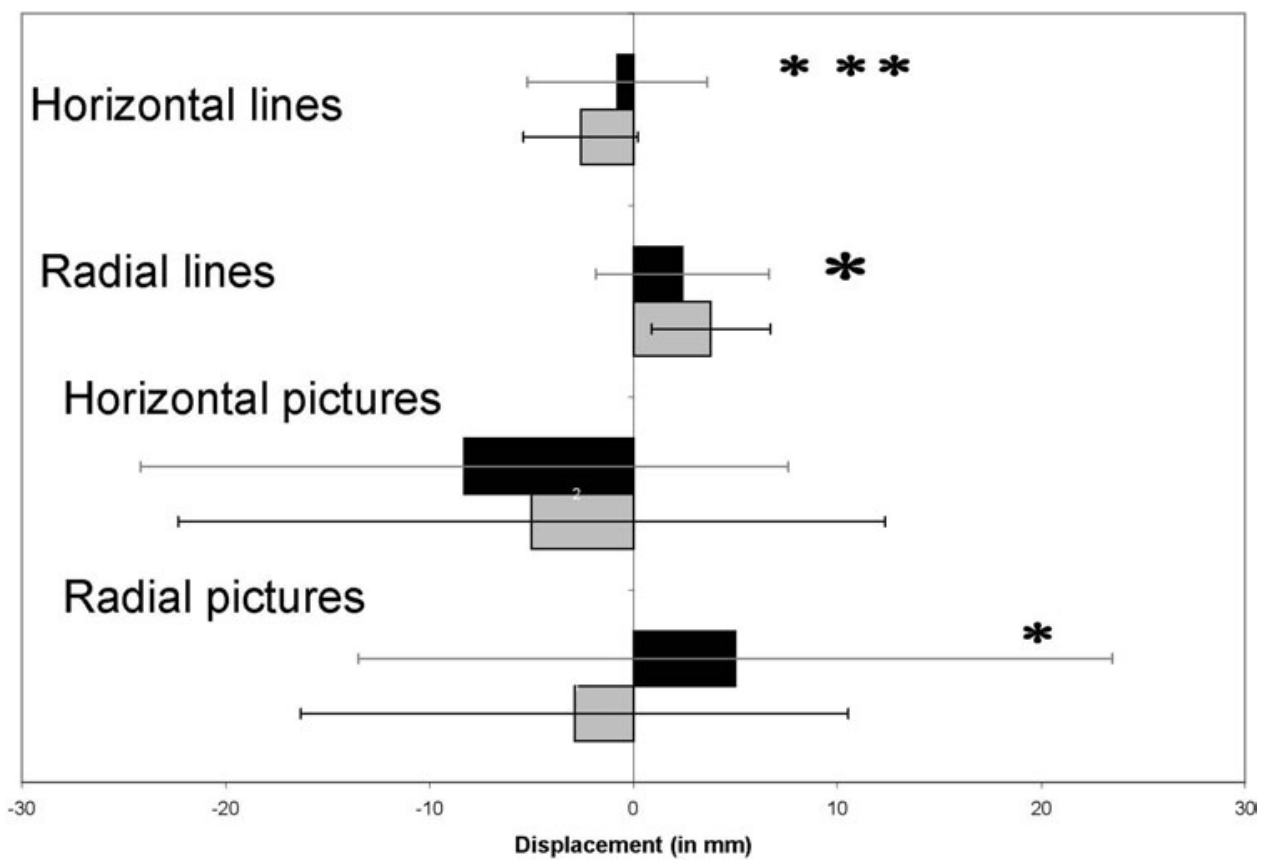

Fig. 4. Results of spatial-syntactic bias assessment in 120 young and aged subjects. The number of sentences (of total 4) drawn with the sentence subject placed on the left of the sentence object appears on the $x$-axis; the number of aged (gray bars with black bars) and young subjects (black bars) appears on the $y$-axis. Although subjects of all ages were left-biased, it can be appreciated that more aged subjects placed the sentence subject on the right (Mann-Whitney $U$; $p<.05)$. See text for details.

subjects did not (mean $-0.59 \mathrm{~mm} ; S D$ 17.47; $t=2.95 ; p=$ $.004)$.

\section{(C) Effects of gender on line bisection}

We had no specific a priori hypotheses about the effects of gender on the visual-spatial or spatial-syntactic tasks, although it is possible that leftward line bisection bias might be larger in magnitude in men than in women (Jewell \& McCourt, 2000). To evaluate whether main effects or interactions involving gender might affect interpretation of our line bisection results, we performed a $2 \times 2 \times 2 \times 2$ multivariate analysis of variance as above (Differences by line length) with an additional between-subjects factor gender (female, male). There was an age by gender interaction $(F=$ 5.24; $p=.024)$, with greater line bisection accuracy in women compared with men, in the aged as compared with the young subjects (aged women: mean $-0.33 \mathrm{~mm}$; SD 2.75; aged men: mean $1.70 \mathrm{~mm}$; $S D$ 3.29; $t=-2.32 ; p=$ .025 ; young women: mean $0.73 \mathrm{~mm}$; $S D$ 1.65; young men: mean $0.50 \mathrm{~mm}$; SD 2.40; $p=.07$; n.s.). No other significant higher level interactions with gender were observed.

\section{(D) Effects of gender on drawing placement bias}

As above, we did not have specific hypotheses about the effect of gender on drawing placement. We performed a $3 \times 2 \times 2 \times 2$ repeated-measures analysis of variance with drawing type (house/tree/person), axis (horizontal vs. radial), age and gender as within and between subjects factors. Results were similar to analysis under C). There was an age by gender interaction $(F=6.52 ; p=.012)$, and it appeared that bias differed in aged men and women, but did not differ in young men and women. The difference in aged subjects was by mean direction of bias rather than by accuracy $($ aged men $=$ mean $2.31 \mathrm{~mm} ; S D$ 6.12; aged women $-5.6 \mathrm{~mm} ; S D$ 8.41; $t=4.12 ; p<.001$; young men $=$ mean $-4.26 \mathrm{~mm}$; $S D$ 11.32; young women mean $-3.92 \mathrm{~mm}$; $S D$ $9.76 ; p=.8 ;$ n.s. $)$. There was a main effect of gender $(F=$ $4.73 ; p=.03)$, attributed to the above interaction. No other higher level interactions with gender were observed.

\section{(E) No detected effect of gender on spatial-syntactic bias}

We performed a Mann-Whitney $U$ comparing the spatialsyntactic ratio between subjects grouped by gender. There was no sex difference observed $(z=-1.36 ; p=.18$, n.s.).

\section{DISCUSSION}

Please see Figure 1, which outlines in diagram format the logic of the present study, some results emphasized below, and possible conclusions. Others reported that, in aged people, leftward horizontal line bisection bias may be reduced (Jewell \& McCourt, 2000). We replicated this finding in a group of 60 aged subjects as compared with a matched 
group of 60 young subjects: aged subjects in our study did not manifest significant leftward or rightward line bisection errors (i.e., were accurate). However, when assessed with an implicit horizontal spatial bias task (placement of a drawing on paper relative to the page center), aged subjects demonstrated leftward bias of comparable magnitude to that observed in young subjects. Explicit far bias on a line bisection task was similarly reduced in this group of aged subjects relative to young subjects, but on an implicit (drawing placement) task, aged subjects actually demonstrated more far bias than did young subjects (whose mean bias was proximal, but not significantly different from perfect performance).

The results above are consistent with a relative loss of hemispheric laterality as dorsal-stream visual system systems are affected by aging. We hypothesized this might not affect radial bias, if left dorsal cortical activation experienced a compensatory increase, thus maintaining or increasing dorsal-ventral visual functional dissociation. We intended to test for maintained dorsal-ventral functional dissociation by evaluating whether far bias was comparable or increased in aged subjects, compared with young subjects. Because we found far bias in aged people was maintained on explicit line bisection (although reduced compared with young subjects) and far bias was increased on an implicit visualspatial task compared with young subjects, it is possible that reduction in hemispheric asymmetry occurs within other, maintained functional cortical networks.

Our findings may be consistent with previous suggestions that hemispheric asymmetry decreases with aging (Cabeza, 2002). The HAROLD model does not discuss distinct activation in dorsal/ventral visual streams, but the current findings suggest a loss of hemisphericity is not a marker for general reduction in functional localization of cognition in aging.

We noted that leftward bias was reduced in aged subjects compared with young subjects when these subjects bisected lines, and when they drew pictures depicting the action of a sentence (an implicit spatial-syntactic task, Chatterjee et al., 1995). This argues that neither performance factors (increased accuracy) nor pure representational properties of drawings versus lines can solely explain age differences in spatial bias. However, because drawings were placed more leftward on a page in aged as compared with young subjects (explicit/implicit dissociation for the two visual-spatial tasks), we cannot rule out a secondary effect on lateral spatial bias of task type (drawing $v s$. making ballistic bisection marks). It is also possible, because far bias may have been more marked in aged subjects in placing house/tree/person drawings as compared with line bisections, and because we could not assess radial bias concurrently with the implicit spatial-syntactic task, that explicit versus implicit performance factors exert a secondary effect on age-related differences in radial bias.

This study did not completely account for possible confounding factors producing leftward bias on the spatialsyntactic task. For example, the current study would be unable to differentiate implicit left-to-right organization of a drawing produced to illustrate the action of a sentence primarily determined by subject-object action from the same implicit spatial organization determined by the order in which the elements are read. Although these may both represent spatial function, the first may be more traditionally syntactic or grammatical than the second. Using passive sentences "The child's hair is combed by the mother" might distinguish these effects. However, in the past, we noted that subjects with average education had difficulty reliably interpreting passive sentences; thus, we elected not to investigate them here. A paradigm including both active and passive sentences would be appropriate to compare with the current findings in follow-up studies.

Young subjects in the current study erred distal to veridical center when bisecting radial lines, as in previous studies (Barrett et al., 2002a; Chewning et al., 1998; Geldmacher \& Heilman, 1994). However, young subjects in the current study did not make significant distal errors when centering house, tree or person drawings on a page. In contrast, aged subject "house" and "tree" drawings were centered distal to page center, while "person" drawings were placed without significant proximal-distal bias. It is possible, as we suggest, that aging may actually increase far spatial bias. If far bias may be more attributable to left hemisphere, dorsal spatial function than to right hemisphere activation, loss of right hemisphere dominance for spatial tasks may actually increase far bias with aging. Why subjects centered "person" drawings more proximally, however, is unclear. It is possible that drawing a person activates egocentric spatial systems strongly associated with near space more than drawing a house or tree, which may activate allocentric systems associated with far spatial regions. Another possibility is that radial placement of person versus tree/house drawings may manifest differences relative to differences in representation based upon mapping vertical gaze direction on a near-far axis. If those objects customarily viewed by upgaze are remapped as "far" on a radial axis, relative to objects customarily viewed at eye level or by downgaze (which may be coded as "near," this could explain our results. Follow-up studies with drawings of other objects associated with up- and down-gaze, such as shoes, clouds, and so on, could elucidate whether this hypothesis contributed to our findings.

Our study has a number of important limitations. We noted that the effects of aging on visual-spatial bias may vary by gender. Alteration of bias with aging appeared more marked in men than in women in this subject group, although this requires further confirmation. We also did not control for other factors which may influence drawing performance or horizontal/radial spatial bias, including educational drawing experience, expectations of what was assessed in implicit tasks, or eye dominance. We only included subjects in this study who reported that they wrote with their right hand, and did not assess degrees of handedness, which is a major limitation to applicability of our results to the general population. Lastly, we did not con- 
sider systematic differences in drawing size, and drawing size/drawing placement relationships in the current study. Larger drawings may be subject to less lateral and radial displacement, but they also may represent more elaborate products of spatial function, qualitatively different than smaller and simplified drawings. Future experiments are needed examining the interaction of aging and other factors affecting spatial bias; examining the mechanisms of age differences for both implicit and explicit horizontal and radial spatial bias, including handedness assessed by sensitive performance measures; and examining whether drawing size, or size-placement relationships, may vary systematically with spatial bias in aging.

\section{ACKNOWLEDGMENTS}

The information in this manuscript, and the manuscript itself, has never been published either electronically or in print. Supported by the National Institutes of Health/National Institute of Neurological Disorders and Stroke (K08NS002085and K02 NS47099, Barrett), and the Henry H. Kessler Foundation (Barrett). Presented in preliminary form at the 34th annual meeting of the International Neuropsychological Society, JINS 2006; 21-22. No financial conflicts of interest apply to this work. We thank the participants in this study for donating their time for testing. Lynn Leidig collected the majority of the data analyzed in this report, assisted by John Garza, Murali Nagarajan, Keri Muniz, Melissa Ingram, Jennifer Mills and Antonee Stern, and Chris Spofford assisted with subject recruitment; we thank all of them for their help, and Lynn Leidig for data summaries and organization, without which this study would not have been possible. We are grateful to Danielle Alexander and Meghan Davis for assisting with data entry and some statistical analyses, and to Anjan Chatterjee for suggesting one of the post hoc analyses upon our preliminary presentation of this data. We thank three anonymous reviewers for valuable help clarifying the presentation and content of this paper based upon review of a preliminary version, one of whom suggested further examination of the influence of eye gaze direction on spatial representations is warranted based on the current results.

\section{REFERENCES}

Barrett, A.M., Crosson, J.B., Crucian, G.P., \& Heilman, K.M. (2002b). Far bias on the radial line bisection task: Measuring perceptual-attentional and motor-intentional bias in normal subjects. Cortex, 38, 769-778.

Barrett, A.M., Kim, M.H., \& Heilman, K.M. (2002a). Spatial bias in Korean right-left, vertical readers. Neuropsychologia, 40, 1003-1012.

Buck, J.N. (1966). The House-Tree-Person Technique-A Revised Manual. Los Angeles: Western Psychological Services.

Cabeza, R. (2002). Hemispheric asymmetry reduction in older adults: The HAROLD model. Psychology and Aging, 17, 85-100.

Chatterjee, A. (2001). Language and space: Some interactions. Trends in Cognitive Sciences, 5, 55-61.

Chatterjee, A., Maher, L.M., \& Heilman, K.M. (1995). Spatial characteristics of thematic role representation. Neuropsychologia, 33, 643-648.
Chewning, J., Adair, J.C., Heilman, E.B., \& Heilman, K.M. (1998). Attentional bias in normal subjects performing visual and tactile radial line bisections. Neuropsychologia, 36, 1097-1101.

Dolcos, F., Rice, H.J., \& Cabeza, R. (2002). Hemispheric asymmetry and aging: Right hemisphere decline or asymmetry reduction. Neuroscience \& Biobehavioral Reviews, 26, 819-825.

Folstein, M.F., Folstein, S.E., \& McHugh, P.R. (1975). "MiniMental State": A practical method for grading the cognitive status of patients for the clinician. Journal of Psychiatric Research, 12, 189-198.

Geldmacher, D.S. \& Heilman, K.M. (1994). Visual field influence on radial line bisection. Brain and Cognition, 26, 65-72.

Geminiani, G., Bisiach, E., Berti, A., \& Rusconi, M.L. (1995). Analogical representation and language structure. Neuropsychologia, 33, 1565-1574.

Harvey, M. \& Butler, S.H. (2004). Aging affects perceptual and eye-movement biases apparent in chimeric face processing. Perception, 33, 106.

Heller, W. (1991). Hemispatial biases in children on the Draw-APerson Test. Developmental Neuropsychology, 7, 151-160.

Jewell, G. \& McCourt, M.E. (2000). Pseudoneglect: A review and meta-analysis of performance factors in line bisection tasks. Neuropsychologia, 38, 93-110.

Jordan, T.J. \& Patching, G.R. (2003). Assessing effects of stimulus orientation on perception of lateralized words and nonwords. Neuropsychologia, 41, 1693-1702.

Kosslyn, S.M., Koenig, O., Barrett, A., Backer Cave, C., Tang, J., \& Gabrieli, J.D.E. (1989). Evidence for two types of spatial representations: Hemispheric specialization for categorical and coordinate relations. Journal of Experimental Psychology: Human Perception and Performance, 15, 723-735.

Mark, V.W. \& Heilman, K.M. (1998). Diagonal spatial neglect. Journal of Neurology, Neurosurgery and Psychiatry, 65, $348-352$.

Milner, A.D. \& Goodale, M.A. (1996). The Visual Brain in Action. (pp. 67-120). Oxford: Oxford University Press.

Previc, F.H. (1990). Functional specialization in the lower and upper visual fields in humans: Its ecological origins and neurophysiological implications. Behavioral and Brain Sciences, 13, 519-542.

Previc, F.H. \& Blume, J.L. (1993). Visual search asymmetries in three-dimensional space. Vision Research, 33, 2697-2704.

Reuter-Lorenz, P.A. \& Posner, M.I. (1990). Components of neglect from right-hemisphere damage: An analysis of line bisection. Neuropsychologia, 28, 327-333.

Tegner, R. \& Levander, M. (1991). The influence of stimulus properties on visual neglect. Journal of Neurology Neurosurgery and Psychiatry, 54, 882-887.

Ungerleider, L.G. \& Mishkin, M. (1982). Two cortical visual systems. In D.J. Ingle, M.A. Goodale, \& R.J.W. Mansfield (Eds.), Analysis of Visual Behavior (pp. 549-586). Cambridge, MA: MIT Press.

Varnava, A., McCarthy, M., \& Beaumont, J.G. (2002). Line bisection in normal adults: Direction of attentional bias for near and far space. Neuropsychologia, 40, 1372-1378.

Weiss, P.H., Marshall, J.C., Zilles, K., \& Fink, G.R. (2003). Are action and perception in near and far space additive or interactive factors? Neuroimage, 18, 837-846. 